\title{
Split-tendon Flexor Pulley Reconstruction with Application of Tendon Rod
}

\author{
Adel Hussein Amr MD; Hesham Aly Helal MD.
}

Plastic Surgery Department, Ain Shams University, Cairo, Egypt.

Purpose: Flexor pulley reconstruction is a challenging surgery. Regardless of the reconstructive technique used, the surgeon should make every effort to imitate the length, tension and glide of the normal pulley. We describe in the present study a new method for pulley reconstruction in using a split tendon graft.

Patients and methods: Twelve adult patients had A2 and/or A4 pulley reconstruction using split-tendon during application of tendon rod as a first stage tendon reconstruction. This was followed by application of an autogenous tendon graft in a second stage 12 weeks from the first stage. Patients were followed for 6 months after the second stage.

Results: All patients recovered a satisfactory range of motion with an average finger to palm distance of $16 \mathrm{~mm}$.

Conclusion: The described technique is versatile and provides an autogenous thin graft that is free of donor site morbidity with excellent tendon gliding.

Key words: Pulley injury, pulley reconstruction, two stage tendon reconstruction, split tendon.

\section{Introduction:}

In the fingers, the flexor tendon courses through a fibrosseous tunnel that is reinforced by the pulley system and lined by a synovial sheath. This sheath forms a closed space providing nutrition and allows smooth gliding of the flexor tendon. ${ }^{1,2}$ In addition the retinacular reinforcing pulleys maintain the flexor tendons close to the volar surface of the phalanges and thus maximizing mechanical efficiency and preventing bowstringing. 3,4

The retinacular pulley system consists of five annular pulleys and three cruciform pulleys. Both A2 and A4 pulleys, located over the proximal and middle phalanges, are usually considered the most important in terms of structural support and mechanical efficiency. 5,6 This functional importance necessitates proper restoration of at least the integrity of these two pulleys after injury to allow early rehabilitation. 7,8

The search for the ideal pulley reconstruction had been a matter of debate throughout the years and various studies addressed two main subjects; the material to be used and the method of fixation. Several authors had suggested various methods for flexor pulley reconstruction. Some did not encircle the phalanx while others looped around the phalanx. A tendon graft looped through the remnant of the injured pulley was used. Others used a slip of the superficialis tendon while its insertion was left intact. A graft of the extensor retinaculum was also sutured to the remnant of the injured pulley or directly to the periosteum. Others used tendon grafts which was looped around the phalanx, using different number of loops depending on the width of the pulley they needed. ${ }^{9-11}$ Still, no single technique has shown superiority over the others concerning the final result. In the present study, we describe a new method for pulley reconstruction using split tendon.

\section{Patients and methods:}

This study was conducted between April 2014 and January 2015 in Ain Shams University Hospitals. 12 patients were 
operated upon. All patients sufferedcomplete loss of A2 pulley or a combined A2 and A4 loss together with neglected or failed flexor tendon injury. They were scheduled to 2 stages tendon reconstruction and pulley reconstruction. The newly described techniquewas performed during the first stage with the application of tendon rod. We excluded patients with crushing injuries and skin loss, injury to the neurovascular bundle and stiff joints. An informed consent was obtained from each patient included in the study.

\section{Surgical technique:}

In all cases, the operation was done under supraclavicular block anesthesia. Tourniquet was inflated to $250 \mathrm{mmHg}$ after exsanguinations of the arm. Exposure of the tendon and pulley system was done through Brunner incision. The state of tendon and pulleys was determined. Upon deciding to reconstruct the pulley system, the fibrosed tendons and pulleys were excised. A tendon rod size 5 was used. It was attached distally to the distal part of the flexor digitorum profundus. Proximally it was left free either in the palm or distal forearm. A healthy part of the excised profundus tendon was cut with a length of $1-1.5 \mathrm{~cm}$ according to the pulley which is to be reconstructed.

It was split in an open book fashion and sutured in place by four $4 / 0$ prolene sutures to the remnants of the original pulley with the outer surface of the tendon facing the tendon rod Figures $(\mathbf{1 , 2})$.

Intra-operative testing for gliding and bowstringing was done, the tourniquet was then deflated and meticulous hemostasis done. Closure was done by $5 / 0$ prolene sutures. Simple, non-bulky dressing was applied. Antibiotics and nonsteroidal antiinflammatory drugs were prescribed for five days post operatively. Patients were instructed to start applying passive range of motion, between 0-45 degrees within three days postoperatively. Sutures were removed two weeks after the surgery. Full passive flexion/ extension was done by the patient several times a day until the date of the second stage. The second stage was performed after 12 weeks provided all joints were lax with full passive range of motion. A tendon graft was harvested (Palmaris Longus) and replaced the tendon rod underneath the reconstructed pulleys. Patients were evaluated by measurement of distance between pulp to palm and range of motion (ROM).

Follow-up examination was performed for all thepatients and included measurement of the ROM of affected digits at 4, 8, 12 and 24 weeks postoperatively using a finger goniometer. The angles of metacarpophalengeal (MCP), proximal interphalengeal (PIP), and distal interphalengeal (DIP) joints were measured with active flexion and extension with the forearm and wrist in a neutral position.

Total Active Motion (TAM) evaluation of the American Society for Surgery of the Hand12 was applied as follows:

Excellent grade $=100 \%$ normal

Good grade $=75-99 \%$ normal

Fair grade $=50-74 \%$ normal

Poor grade $=<50 \%$ normal

\section{Statistical analysis}

Statistical package for the social sciences program was used. Continuous variables are expressed as mean $\pm \mathrm{SD}$ or as median (interquartile range) in cases of skewed distributions. Categorical variables are expressed as frequencies and percents.

\section{Results:}

12 patients were operated upon for reconstruction of A2 and/or A4 flexor pulleys. Nine patients suffered from neglected flexor tendon injuries for more than 2 months while the other 3 patients had failed primary repair and tenolysis.

Eight patients $(67 \%)$ had a right hand injury while the other four (33\%) had a left hand injury (all patients had a dominant right hand); among these, two index (17\%), four middle (33\%), five ring (42\%) and one little $(8 \%)$ digits were affected.

Ages ranged between 18 and 42 years $[$ mean $=27.0714$, S.D $=5.48374]$, male: female ratio was 9:3. The patients were examined periodically in the follow up period 

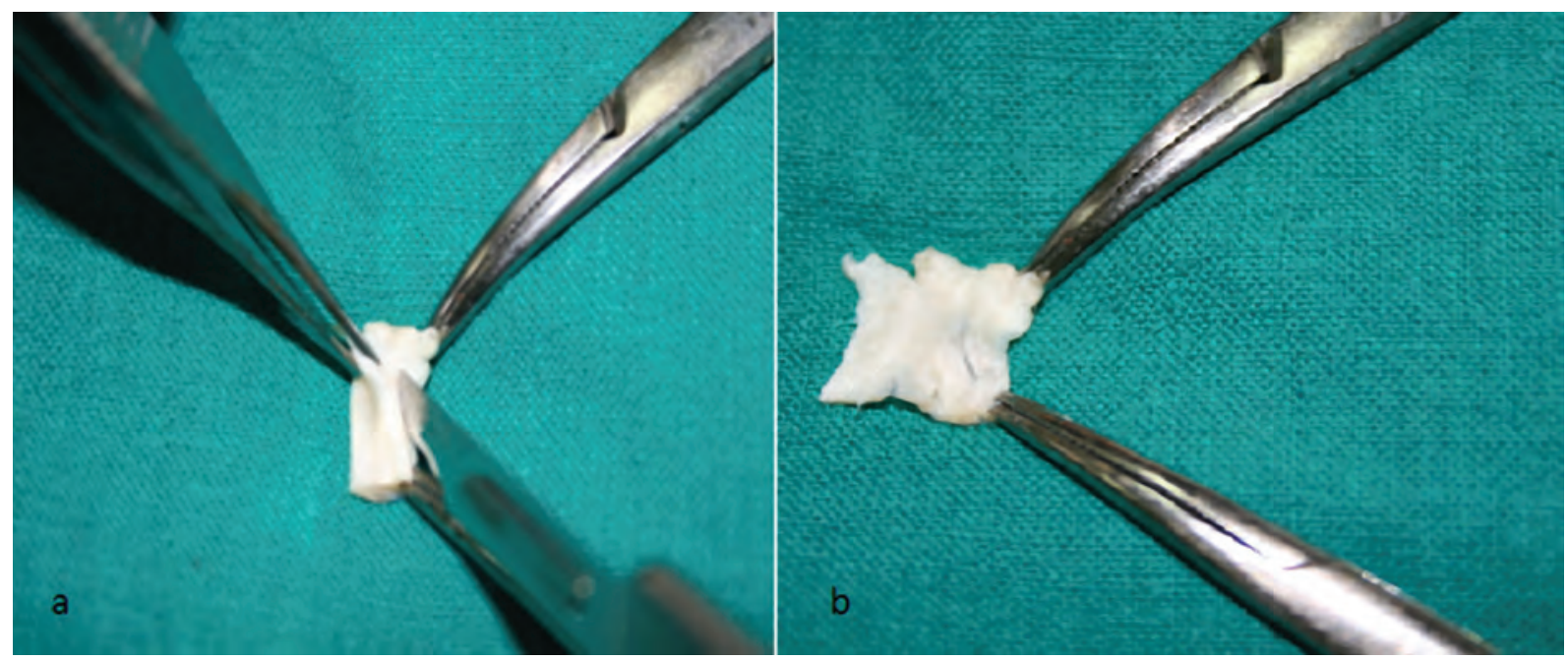

Figure (1): Splitting of the tendon graft.
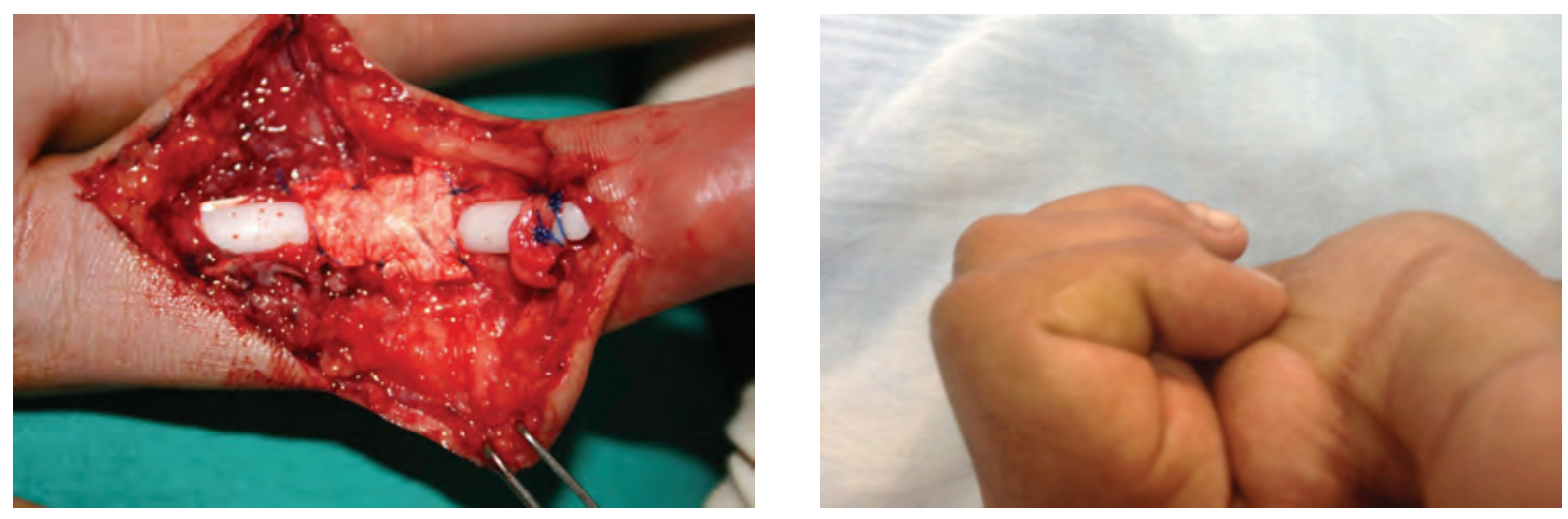

Figure (2): Intra-operative photograph showing sutured graft as A2 and A4 pulleys.

from the first till the second stage and then at 4, 8, 12 and 24 weeks after the second stage.

The pulp to palm distance was $<10 \mathrm{~mm}$ in 3 patients and 10-20 $\mathrm{mm}$ in 9 patients with an average distance of $16 \mathrm{~mm}$ Figure (3). No patients required a tenolysis and also no one had a secondary pulley rupture.

At the 6-month final follow-up evaluation, the MCP hyperextension deficit remained at $20^{\circ}$, with the following active ranges of motion: $\mathrm{MCP}, 0^{\circ}-103^{\circ}$; PIP, $10^{\circ}-110^{\circ}$; DIP, $0^{\circ}-80^{\circ}$. Average active Range of motion: MP 90 degrees/ PIP 95 degrees/ DIP 70 degrees.

According to the American Society for Surgery of the Hand Score, the TAM was poor grade in 1 patient, fair grade in 3 patients and good grade in 8 patients.

\section{Discussion:}

Plenty of techniques and options regarding methods of flexor tendon pulley

Figure (3): Active motion of the ring finger at 6 months post-operative.

reconstruction, whether primary repair of pulley alone or combined with tendon reconstruction, have been described.Most of these studies have addressed two main problems; the source of the graft and its method of fixation.

In the present study we describe a new technique for the reconstruction of the flexor pulley during first stage tendon reconstruction in twelve patients with the application of tendon rod.

As the usage of grafts in synovial lined sheaths was found to be more superior over extrasynovial grafts based on studies concerned with the testing of biomechanics of the tendon function; ${ }^{13-16}$ we used split remnants of the flexor digitorum profundus tendon for replicating the A2 and/or A4 pulleys. The splitting of the tendon gave us the advantages of the autogenous intrasynovial graft as no donor site morbidity, no foreign 
body reaction, a better gliding of the tendon with less resistance. In addition it provided a thin graft in contrast to many other studies.

On the other hand, two main techniques have been proposed for pulley reconstruction: suturing the graft through the remaining pulley rim, or encircling the bone with the graft making a loop. The around-bone technique can, in some cases, create focal bony ischemia and result in late phalangeal fracture, but it was found mechanically more effective in cadaveric studies. ${ }^{17,18-20}$

In the present study we fixed the graft to the remnant of the injured pulley, trying to perform amore anatomical reconstruction and provide no interference with the extensor mechanism with less dissection and avoiding the possible complications of the around bone technique even if the later was found to be mechanically effective in cadaveric studies. ${ }^{18-20}$

Many authors have noticed recurrence of the bowstringing as a result of stretching out of the graft by time and thus they advocated the usage of synthetic grafts as silicon, Dacronand polytetrafluroethylene. ${ }^{21}$ This was not the case in the present study, 11 patients showed good range of motion with no bowstringing at the end of the follow-up period.

The described technique is versatile and provides an autogenous thin graft that is free of donor site morbidity with long standing excellent tendon gliding.

\section{Reference:}

1- Tanaka T, Amadio PC, Zhao C, Zobitz ME, An KN: The effect of partial A2 pulley excision on gliding resistance and pulley strength in vitro. J Hand Surg 2004; 29A: 877-883.

2- Naidu SH, Rinkus K: Multiple-loop, uniformtension flexor pulley reconstruction. $J$ Hand Surg 2007; 32A: 265-268.

3- Strauch B, De Moura W: Digital flexor tendon sheath: An anatomic study. J Hand Surg 1985; 10A: 785.

4- Rispler D, Greenwald D, Shumway S, Allan C, Mass D: Efficiency of the flexor tendon pulley system in human cadaver hands. $J$ Hand Surg Am 1996; 21: 444-450.

5- Peterson WW, Manske PR, Bollinger BA,
Lesker PA, McCarthy JA: Effect of pulley excision on flexor tendon biomechanics. $J$ Orthop Res 1986; 4: 96-101.

6- Doyle JR: Anatomy of the finger flexor tendon sheath and pulley system. $J$ Hand Surg 1988; 13A: 473.

7- Lourie GM, Hamby Z, Raasch WG, Chandler JB, Porter JL: Annular flexor pulley injuries in professional baseball pitchers: A case series. Am J Sports Med 2011; 39: 421-424.

8- Arora R, Fritz D, Zimmermann R, Lutz M, Kamelger F, Klauser AS, Gabl M: Reconstruction of the digital flexor pulley system: A retrospective comparison of two methods of treatment. J Hand Surg Eur 2007; 32: 60-66.

9- Clark TA, Skeete K, Amadio PC: Flexor tendon pulley reconstruction. $J$ Hand Surg 2010; 35A: 1685-1689.

10- Bakhach J, Sentucq-Rigal J, Mouton P, Boileau R, Panconi B, Guimberteau JC: The Omega "Omega" pulley plasty. A new technique to increase the diameter of the annular flexor digital pulleys. Ann Chir Plast Esthet 2005; 50: 705-714.

11- Bunata RE: Primary pulley enlargement in zone 2 by incision and repair with an extensor retinaculum graft. J Hand Surg Am 2010; 35 : 785-790.

12- Hung LK, Pang KW, Yeung PL, Cheung L, Wong JM, Chan P: Active mobilization after flexor tendon repair: Comparison of results following injuries in zone 2 and other zones. J Orthop Surg 2005; 13: 158-163.

13- Tang JB, Zhang QG, Ishii S: Autogenous free sheath grafts in reconstruction of injured digital flexor tendon sheath at the delayed primary stage. J Hand Surg Br 1993; 18: 31-32.

14- Odobescu A, Radu A, Brutus JP, Gilardino MS: Modified flexor digitorum superficialis slip technique for A4 pulley reconstruction. $J$ Hand Surg Eur 2010; 35: 464-468.

15- Nishida J, Amadio PC, Bettinger PC, An KN: Flexor tendon-pulley interaction after pulley reconstruction: A biomechanical study in a human model in vitro. J Hand Surg Am1998; 23: 665-672.

16- Seiler JG, Uchiyama S, Ellis F, Amadio PC, Gelberman RH, An KN: Reconstruction of the flexor pulley. The effect of the tension and source of the graft in an in vitro dog model. $J$ Bone Joint Surg Am 1998; 80: 699-703.

17- Metha V, Phillips CS: Flexor tendon pulley reconstruction. Hand Clin 2005; 21: 245-251. 
18- Karev A,Stahl S, Taran A: The mechanical efficiency of the pulley system in normal digits compared with a reconstructed system using the "belt loop" technique. J Hand Surg 1987; 12A: 596-601.

19- Widstrom CJ, Johnson G, Doyle JR, Manske PR, Inhofe P: A mechanical study of six digital pulley reconstruction techniques: Part I. Mechanical effectiveness. J Hand Surg
1989; 14A: 821-825.

20- Widstrom CJ, Doyle JR, Johnson G, Manske PR, McGee R. A mechanical study of six digital pulley reconstruction techniques: Part II. Strength of individual reconstructions. $J$ Hand Surg 1989; 14A: 826-829.

21- Dy CJ, Daluiski A: Flexor Pulley Reconstruction. Hand Clin 2013; 29(2): 235-242. 